\title{
Controlling the Spectrum of Spontaneous Parametric Down- Conversion in Nonlinear Crystals by External Electric Field
}

\author{
D.O. Akatiev ${ }^{1 \mathrm{a}}$ and A.A. Kalachev ${ }^{1,2}$ \\ ${ }^{1}$ Kazan Federal University, 18 Kremlevskaya str. Kazan 420000, Russia \\ ${ }^{2}$ Zavoisky Physical-Technical Institute RAS, 10/7 Sibirsky tract str., Kazan 420029, Russia
}

\begin{abstract}
We discuss the possibilities of controlling the spectrum of correlated photons generated via spontaneous parametric down-conversion by using external homogeneous electric field acting on the nonlinear crystal.
\end{abstract}

Keywords: single-photon states, spontaneous parametric down-conversion

The phenomenon of spontaneous parametric down-conversion (SPDC) has been widely used in modern quantum optics and information technology for preparing various nonclassical states of the electromagnetic field and for correlation measurements. In particular, the development of effective sources of single-photon states is of practical importance [1]. The phenomenon of SPDC allows one to generate correlated photon pairs and single photons in a broad spectral range and prepare pure singlephoton states at room temperature. For various quantum information applications the interaction of single photons with resonance atomic systems (e.g., quantum memory devices) is demanded. In this case, when the photon spectral bandwidth is of the order of the absorption line, the frequency stabilization of a single-photon source under changing external conditions (non-stable wavelength of the pump, non-stable temperature, etc.) proves to be crucial.

In the present work, we study the possibilities of controlling the SPDC spectrum by using external homogeneous electric field acting on the nonlinear crystal. The method is based on the electro-optic effect (Pockels effect), wherein the external electric field changes the refractive index indicatrix of the nonlinear crystal, which leads to a frequency shift of the SPDC spectrum [2]. By tracking changes of the refractive index indicatrix it is possible to estimate the necessary frequency shift and compensate the instability of the pump laser field and other effects. In the course of the present work we focus on a promising regime of SPDC with photons emitted in opposite directions, which is possible in periodically-poled nonlinear crystals and waveguides [3].

The work is supported by the Russian Science Foundation (Grant No. 14-12-00806).

\section{References}

1. M.D. Eisaman et al. // Review of Scientific Instruments. 82. P. 071101 (2011)

2. K.G. Katamadze et al. // JETP Lett. 94. P. 284 (2011)

3. A. A. Shukhin, et al. // J. Phys.: Conf. Ser. 613. P. 012015 (2015)

${ }^{\text {a } C o r r e s p o n d i n g ~ a u t h o r: ~ a k a t e v d m i t r i j j @ g m a i l . c o m ~}$

This is an Open Access article distributed under the terms of the Creative Commons Attribution License 4.0, which permits unrestricted use, distribution, and reproduction in any medium, provided the original work is properly cited. 